\title{
Load Characterization in Medium Voltage of an Electric Distribution Utility Related to Active Filters
}

\author{
F. Corasaniti (*), Student Member, IEEE, M. B. Barbieri (*), Senior Member, IEEE, P. Arnera (*), \\ Senior Member, IEEE, and M. I. Valla (**), Senior Member, IEEE
}

\begin{abstract}
The behavior of the actual load of an electric energy distribution utility by means of voltage and current measurements is analyzed in this paper. Steady and transient states are taken into account. The main attention is fixed in points where present shunt compensation is installed. Results of field measurements and the connection and disconnection of capacitor banks are shown. Studies for future compensation are detailed and the possible advantages of active filter incorporation are analyzed in order to replace or complement the existing compensation. The minimal requirements are established in order to consider the design of the active filters and their compatibility with the existing installations.
\end{abstract}

Index terms: Active Filters, Compensation, Harmonic Studies, Measurements.

\section{INTRODUCTION}

$\mathrm{T}_{\mathrm{p}}^{\mathrm{h}}$ he increase of non-linear loads and equipments in the power systems has been demanding the compensation of disturbances caused by them. Voltage distortion due to current harmonics is becoming a major problem for the utilities at distribution levels. Utilities frequently encounter harmonic related problems, such as higher transformer and line losses, reactive power, and resonance problems, required de-rating of distribution equipment, harmonic interactions between the utility and loads, reduced system stability and reduced safe operating margins.

Provided the necessity of the reactive compensation in a network of an electric energy distribution utility, measurements were performed at different points of interest in the network in order to carry out harmonic studies. Harmonic and transient measurements were carried out in three different distribution substations where reactive compensation is required. The measurements were carried out in medium voltage levels (MV) where at present there is shunt compensation. The aim was to obtain load characteristics through measurements of voltage and current harmonics at key points.

The harmonic studies are performed considering the future configuration of the network. First, future compensation with capacitor banks is analyzed [2]. Voltage distortions in different points of the network [5] and the working conditions of the capacitor banks [6] are verified by means of harmonic flows.

(*) F. Corasaniti, M.B. Barbieri, P. Arnera are with IITREE-LAT. Facultad de Ingeniería Universidad Nacional de La Plata (FI-UNLP). (1900) 48 y 116. La Plata. Argentina (e-mail: iitree@iitree-unlp.org.ar).

(**) M.I.Valla is with CONICET and LEICI. Facultad de Ingeniería Universidad Nacional de La Plata (FI-UNLP). (1900) 48 y 116. La Plata. Argentina (e-mail: m.i.valla@ieee.org).

1-4244-0288-3/06/\$20.00 @2006 IEEE
Second, the advantages of carrying out the compensation with active filter type equipment or its combination with the traditional compensation are analyzed [1]-[2]. The transient requirements imposed to the active filter are taken into consideration in its design [3]-[4].

\section{Network Description AND Measurements}

Fig. 1 shows the one line diagram of the transmission network of the electric distribution utility. The $132 \mathrm{kV}$ network where the distribution substations (DS) under study are connected works meshed and connected to the $500 \mathrm{kV}$ high voltage transmission system, through two points.

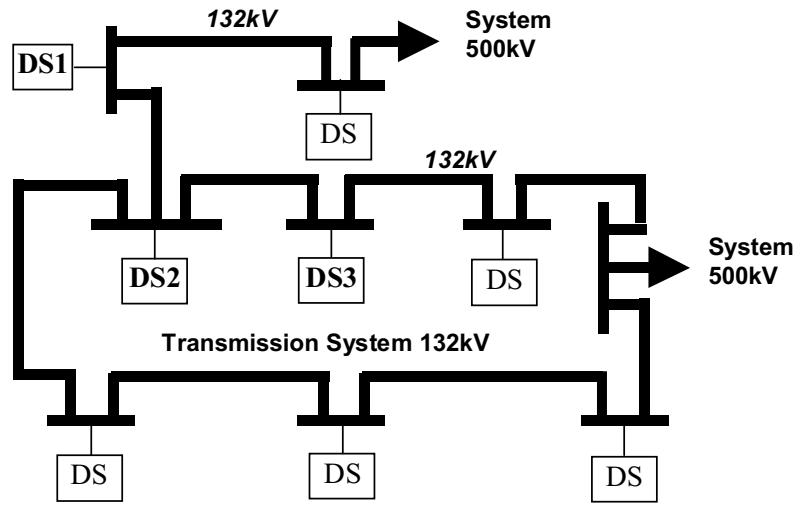

Fig. 1. One line diagram of actual network distribution utility.

The field measurements were carried out in the three DS where additional compensation will be required by the network reconfiguration (DS1 DS2 DS3). Harmonic levels of voltage and currents were recorded along one week.

Voltages and currents were also measured during connection and disconnection of the present capacitor banks in order to observe the transients produced by such switching.

\section{A. Characteristics of DS1.}

Fig. 2 shows the one line diagram of DS1.

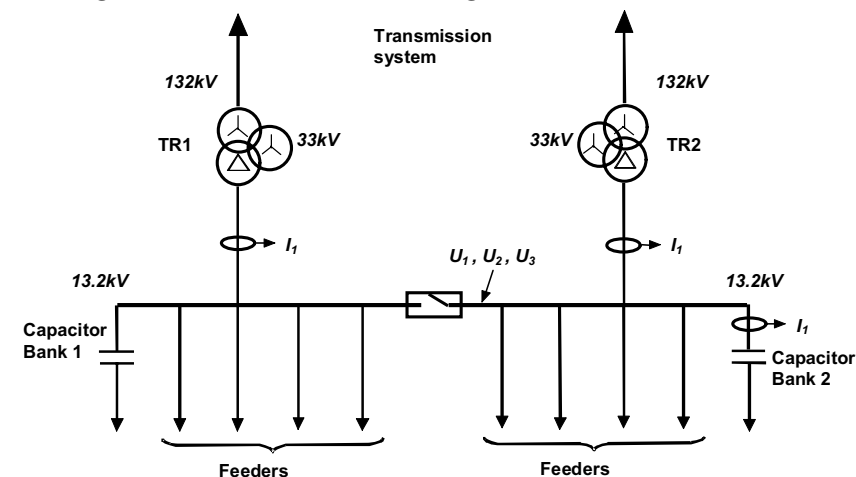

Fig. 2. One line diagram of DS1 and recorded magnitudes. 